\title{
SPECTRAL SYNTHESIS OF FUNCTIONS OF BOUNDED VARIATION
}

\author{
AHARON ATZMON
}

ABSTRACT. It is proved that every bounded measurable function on $(-\infty, \infty)$ which for some constant $a>0$ is of bounded variation on $(-\infty$, $-a)$ and on $(a, \infty)$, admits spectral synthesis in the weak-star topolog: of $L^{\infty}(-\infty, \infty)$.

Introduction. Let $\mathbf{R}$ denote the real line and let $\phi \in L^{\infty}(\mathbf{R})$. For every real number $h$, the $h$-translate of $\phi$ is the function $\phi_{h}$ defined by $\phi_{h}(x)=$ $\phi(x-h), x \in \mathbf{R}$. We recall that the weak-star spectrum of $\phi$, denoted by $\mathrm{Sp} \phi$, is the set of real numbers $\lambda$ such that the function $e^{i \lambda x}$ is in the weak-star closed subspace of $L^{\infty}(\mathbf{R})$ spanned by the translates of $\phi$. One says that $\phi$ admits spectral synthesis (in the weak-star topology) if $\phi$ belongs to the weak-star closure of the subspace spanned in $L^{\infty}(\mathbf{R})$ by the functions $e^{i \lambda x}, \lambda \in \operatorname{Sp} \phi$.

The well-known theorem of P. Malliavin [3] proves that weak-star synthesis in $L^{\infty}(\mathbf{R})$ is not always possible. On the other hand it is well known (see [4]) that the following classes of functions in $L^{\infty}(\mathbf{R})$ admit spectral synthesis:

(1) almost periodic continuous functions on $\mathbf{R}$ (which admit spectral synthesis even in the norm topology of $L^{\infty}(\mathbf{R})$ ),

(2) Fourier-Stieltjes transforms of bounded Borel measures on $\mathbf{R}$,

(3) functions in $L^{\infty}(\mathbf{R})$ whose spectrum has a countable boundary.

In this paper we prove the possibility of spectral synthesis for some other classes which are described in the following theorems:

Theorem 1. Let $\phi$ be a bounded measurable function on $(-\infty, \infty)$ which for some constant $a>0$ is of bounded variation on $(-\infty,-a)$ and on $(a, \infty)$. Then $\phi$ admits spectral synthesis.

Corollary. Let $\phi$ be a bounded measurable function on $(-\infty, \infty)$ and

Received by the editors December 7, 1973.

AMS (MOS) subject classifications (1970). Primary 43A45; Secondary 42 A08. 
$a>0$ a constant. Assume that $\phi$ is monotonic on $(-\infty,-a)$ and on $(a, \infty)$. Then $\phi$ admits spectral synthesis.

Theorem 2. Let $\phi$ be a bounded measurable function on $(-\infty, \infty)$ and $E$ a countable closed subset of $\mathbf{R}$. Assume that the following conditions are satisfied:

$$
\begin{gathered}
\sup _{-\infty<A<B<\infty}\left|\int_{A}^{B} e^{-i \lambda x} \phi(x) d x\right|<\infty \quad \text { for every real } \lambda \notin E . \\
\lim _{L \rightarrow \pm \infty}|\phi(L+u)-\phi(L)|=0 \quad \text { for a.e. } u \in \mathbf{R} .
\end{gathered}
$$

Then $\phi$ admits spectral synthesis.

Corollary. Let $\phi$ be a bounded measurable function on $(-\infty, \infty)$ which satisfies condition (1). Assume that $\lim _{x \rightarrow \infty} \phi(x)$ and $\lim _{x \rightarrow-\infty} \phi(x)$ exist. Then $\phi$ admits spectral synthesis.

Proofs of theorems. We show first that Theorem 2 implies Theorem 1.

Let $\phi$ and $a$ be as in Theorem 1, and let $\lambda \neq 0$. For any interval $(c, d)$ disjoint from $(-a, a)$ we get, by integrating by parts:

$$
\int_{c}^{d} e^{-i \lambda x} \phi(x) d x=\frac{-1}{i \lambda}\left[\phi(d) e^{-i \lambda d}-\phi(c) e^{-i \lambda c}-\int_{c}^{d} e^{-i \lambda x} d \phi(x)\right] .
$$

Therefore, denoting by $\|\phi\|_{\infty}$ the $L^{\infty}$ norm of $\phi$ and by $V(I)$ the variation of $\phi$ over an interval $l$, we have:

$$
\begin{aligned}
\left|\int_{c}^{d} e^{-i \lambda x} \phi(x) d x\right| & \leq \frac{1}{|\lambda|}\left(2\|\phi\|_{\infty}+V(c, d)\right) \\
& \leq \frac{1}{|\lambda|}\left(2\|\phi\|_{\infty}+V(-\infty,-a)+V(a, \infty)\right),
\end{aligned}
$$

and since $\left|\int_{-a}^{a} \phi(x) e^{-i \lambda x} d x\right| \leq 2 a\|\phi\|_{\infty}, \phi$. satisfies condition (1).

The fact that $V(-\infty,-a)$ and $V(a, \infty)$ are finite implies that $\phi$ satisfies condition (2). Consequently Theorem 2 implies Theorem 1.

For $f \in L^{1}(\mathbf{R})$ we denote its Fourier transform by $\hat{f}$ defined by

$$
\hat{f}(x)=\int_{-\infty}^{\infty} f(t) e^{-i x t} d t, \quad-\infty<x<\infty,
$$

and for $f \in L^{1}(\mathbf{R})$ and $\psi \in L^{\infty}(\mathbf{R})$ we denote their convolution by $f * \psi$.

For the proof of Theorem 2 we need two lemmas.

Lemma 1. Let $\psi$ be a bounded measurable function on $(-\infty, \infty)$ and $\lambda \neq 0$ a real number: Assume that $\psi$ satisfies the following conditions: 
(3)

$$
\sup _{-\infty<A<B<\infty}\left|\int_{A}^{B} e^{-i \lambda x} \psi(x) d x\right|<\infty
$$

(4)

$$
\lim _{L \rightarrow \pm \infty}|\psi(L+u)-\psi(L)|=0 \quad \text { for a.e. } u \in \mathbf{R} .
$$

Let $f \in L^{1}(\mathbf{R})$ and assume that

$$
\hat{f}(0)=\hat{f}(\lambda)=0
$$

Then

$$
\lim _{L \rightarrow \infty} \int_{-L}^{L}(f * \psi)(x) e^{-i \lambda x} d x=0
$$

Proof. For every $L>0$ it will be convenient to define the following three functions:

$$
\begin{gathered}
S_{L}(t)=\int_{-L-t}^{L-t} \psi(u) e^{-i \lambda u} d u, \quad P_{L}(t)=\int_{L-t}^{L} \psi(u) e^{-i \lambda u} d u, \\
Q_{L}(t)=\int_{-L-t}^{-L} \psi(u) e^{-i \lambda u} d u .
\end{gathered}
$$

Using Fubini's theorem we get, for every $L>0$,

$$
\int_{-L}^{L}(f * \psi)(x) e^{-i \lambda x} d x=\int_{-\infty}^{\infty} f(t) e^{-i \lambda t} S_{L}(t) d t
$$

Hence, taking into account (5) and the fact that $S_{L}(t)-S_{L}(0)=Q_{L}(t)-$ $P_{L}(t)$, we infer that

$$
\int_{-L}^{L}(f * \psi)(x) e^{-i \lambda x} d x=\int_{-\infty}^{\infty} f(t) e^{-i \lambda t} Q_{L}(t) d t-\int_{-\infty}^{\infty} f(t) e^{-i \lambda t} P_{L}(t) d t .
$$

The lemma will be established by showing that

$$
\lim _{L \rightarrow \infty} \int_{-\infty}^{\infty} f(t) e^{-i \lambda t} P_{L}(t) d t=0
$$

and

$$
\lim _{L \rightarrow \infty} \int_{-\infty}^{\infty} f(t) e^{-i \lambda t} Q_{L}(t) d t=0
$$

To prove (7), consider for every $L>0$ the function

$$
g_{L}(t)=\int_{L-t}^{L}[\psi(u)-\psi(L)] e^{-i \lambda u} d u .
$$

It follows from (3) that

$$
\sup _{L>0}\left\|g_{L}\right\|_{\infty}=\sup _{L>0} \sup _{-\infty<t<\infty}\left|g_{L}(t)\right|<\infty .
$$


Changing variables we get that

$$
g_{L}(t)=e^{-i \lambda(L-t)} \int_{0}^{t}[\psi(L+u-t)-\psi(L)] e^{-i \lambda u} d u
$$

and therefore Lebesgue's dominated convergence theorem implies in virtue of (4) that

$$
\lim _{L \rightarrow \infty} g_{L}(t)=0
$$

for every $-\infty<t<\infty$.

Applying the dominated convergence theorem once again, we infer from (9) and (10) that

$$
\lim _{L \rightarrow \infty} \int_{-\infty}^{\infty} f(t) e^{-i \lambda t} g_{L}(t) d t=0
$$

Noticing that

$$
P_{L}(t)=g_{L}(t)+\left(e^{-i \lambda L} / i \lambda\right)\left(e^{-i \lambda t}-1\right)
$$

and using (5) we get that

$$
\int_{-\infty}^{\infty} f(t) e^{-i \lambda t} P_{L}(t) d t=\int_{-\infty}^{\infty} f(t) e^{-i \lambda t} g_{L}(t) d t
$$

which by (11) implies (7). The proof of (8) is similar, and the lemma is established.

Lemma 2. Let $\psi \in L^{\infty}(\mathbf{R})$ and let $E$ be a closed subset of $\mathbf{R}$. Then $\mathrm{Sp} \psi \subset E$ if and only if $\lim _{h \rightarrow 0} \int_{-\infty}^{\infty} \psi(x)((\sin h x) / h x)^{2} e^{-i \lambda x} d x=0$ for every real $\lambda \notin E$.

Proof. The proof of the lemma is included in the proof of Theorem 4.1 in [4].

Proof of Theorem 2. Let $\phi$ and $E$ be as in Theorem 2. To show that $\phi$ admits spectral synthesis we have to prove that for every function $f \epsilon$ $L^{1}(\mathbf{R})$ such that

$$
\hat{f}(t)=0 \text { for } t \in \mathrm{Sp} \phi
$$

we have

$$
\int_{-\infty}^{\infty} f(y) \overline{\phi(y)} d y=0
$$

(See [2, p. 170].)

Let $\psi$ be the function defined by $\psi(x)=\overline{\phi(-x)}$. Theorem 2 will be established by showing that for every function $f \in L^{1}(\mathbf{R})$ which satisfies (12) we have 


$$
f * \psi(x)=0 \quad \text { for every }-\infty<x<\infty
$$

To prove (14) assume first that $f$ satisfies, together with $(12), \hat{f}(0)=0$. It follows from (1) and (2) that

$$
\lim _{L \rightarrow \pm \infty}|\psi(L+u)-\psi(L)|=0 \quad \text { for a.e. } u \in \mathbf{R}
$$

and that for every $\lambda \notin E$

$$
\sup _{-\infty<A<B<\infty}\left|\int_{A}^{B} \psi(u) e^{-i \lambda u} d u\right|<\infty .
$$

Therefore if $\lambda \notin E \cup\{0\}$ and $\lambda \in \mathrm{Sp} \phi$, Lemma 1 implies that

$$
\lim _{L \rightarrow \infty} \int_{-L}^{L}(f * \psi)(x) e^{-i \lambda x} d x=0
$$

and by the known theorem about the regularity of the $(R, 2)$ summability method for integrals $[1$, p. 301] this implies that

$$
\lim _{h \rightarrow 0} \int_{-\infty}^{\infty}(f * \psi)(x)\left(\frac{\sin b x}{b x}\right)^{2} e^{-i \lambda x} d x=0 .
$$

On the other hand, if $\lambda \notin \mathrm{Sp} \phi$ then $\lambda \notin \mathrm{Sp} \psi$ ( since $\mathrm{Sp} \phi=\mathrm{Sp}_{\mathrm{p}}$ ) and therefore $\lambda \notin \mathrm{Sp}_{\mathrm{p}}(f * \psi)$, so that by Lemma 2 condition (15) holds again. Consequently (15) holds for every $\lambda \notin E \cup\{0\}$ and using Lemma 2 again we conclude that $\mathrm{Sp}(f * \psi) \subset E \cup\{0\}$.

On the other hand, the fact that $\hat{f}$ vanishes on $\operatorname{Sp} \phi$ implies that $\mathrm{Sp}_{\mathrm{p}}(f * \psi)$ is perfect (see $[2, \mathrm{p} .172]$ ) and since $E$ is countable, $\mathrm{Sp}(f * \psi)$. is empty, so that by the dual form of Wiener's general Tauberian theorem (see [4, p. 504]), we infer that $f * \psi$ vanishes almost everywhere.

Remembering that the convolution of an $L^{1}$ function with an $L^{\infty}$ function is continuous we conclude that $f * \psi$ vanishes everywhere. This proves (14) under the additional condition $\hat{f}(0)=0$, and therefore Theorem 2 is established in the case $0 \in \mathrm{Sp} \phi$.

To complete the proof, assume that $0 \notin$ Sp $\phi$. Let $g$ be a function in $L^{1}(\mathbf{R})$ such that $\hat{g}$ has compact support disjoint from $\mathrm{Sp} \phi$, and $\hat{g}(0)=1$. By the previous part of the proof, $(f-f * g) * \psi=0$, and by the assumption on the support of $\hat{g}$ we also have $f * g * \psi=0$ (see [2, Theorem 6.1, p. 170]); therefore $f * \psi=0$, and the proof of Theorem 2 is complete.

Examples. We give first an example of a function which satisfies the hypotheses of Theorem 1 and which does not belong to one of the classes of synthesizable functions described in the introduction. 
Let $E$ be a totally disconnected perfect set contained in the interval $(1,2)$, and let $k$ be a $C^{\infty}$-function such that $k^{-1}(0) \cap(1,2)=E$ and $k(0)=1$. Let $K$ be the Fourier transform of $k$ and define $\phi$ by $\phi(x)=\int_{-\infty}^{x} K(t) d t$, $-\infty<x<\infty$. The hypotheses on $k$ imply that $K \in L^{1}(\mathbf{R})$ and therefore $\phi$ is of bounded variation on $\mathrm{R}$. Since $\lim _{x \rightarrow-\infty} \phi(x)=0, \phi$ is not almost periodic. It is easy to see that the Fourier transform of $\phi$ in the sense of distribution is the distribution $\hat{\phi}$ which is defined in the principal value sense by the function $h(x)=i k(x) / x, x \neq 0$; therefore since $k(0)=1, \hat{\phi}$ is not a bounded measure, that is $\phi$ is not a Fourier-Stieltjes transform. Finally, taking into account the fact that $\operatorname{Sp} \phi=\operatorname{support} \hat{\phi}$ (see [2, p. 170]) we get that $\operatorname{Sp} \phi=$ support $k$, and therefore, by the condition on $k^{-1}(0)$, we have $E \subset \operatorname{bdry}(\operatorname{Sp} \phi)$. Consequently, $\phi$ does not belong to the third class of synthesizable functions either.

We conclude by giving two examples of functions which satisfy the hypotheses of Theorem 2, without necessarily satisfying the conditions of Theorem 1.

Let $\phi$ be a continuous function on $\mathbf{R}$ such that $\lim _{x \rightarrow-\infty} \phi(x)=0$ and assume also that $\phi$ satisfies condition (1) of Theorem 2 .

Let $\psi$ be a bounded continuous function on $\mathbf{R}$ with countable spectrum $\left\{\lambda_{n}\right\}_{n=1}^{\infty}$. Each of the following two conditions implies that the function $\phi \cdot \psi$ satisfies the hypotheses of Theorem 2, and therefore admits spectral synthesis:

(1) $\Sigma_{\lambda_{n} \neq 0} 1 / \lambda_{n}^{2}<\infty$.

(2) The set $\left\{\lambda_{n}\right\}_{n=1}^{\infty}\{0\}$ is independent over the rationals. We omit the proofs.

Added in proof. Professor Y. Domar proved (personal communication) that condition (2) in the hypotheses of Theorem 2 can be deleted without affecting the conclusion of the theorem.

\section{REFERENCES}

1. G. H. Hardy, Divergent series, Clarendon Press, Oxford, 1967.

2. Y. Katznelson, An introduction to harmonic analysis, Wiley, New York, 1968. MR 40 \# 1734.

3. P. Malliavin, Surl'impossibilitéde la synthèse spectrale sur la droite, C. R. Acad. Sci. Paris 248 (1959), 2155-2157. MR 21 \#5854a.

4. H. Pollard, The harmonic analysis of bounded functions, Duke Math. J. 20 (1953), 499-512. MR 15, 215.

UNIVERSITY OF PARIS-SUD, MATHÉMATIQUES 425, 91405 ORSAY, FRANCE

Current address: Department of Mathematics, Technion, Haifa, Israel 